\title{
Sylvie FAINZANG
}

Anthropologue, spécialisée dans le domaine de la santé.

Directeur de recherche à l'Inserm et membre du CERMES

Centre de Recherche Médecine, Sciences, Santé et Société)

(1999)

\section{"La construction culturelle de la norme et de la pathologie"}

Un document produit en version numérique par Jean-Marie Tremblay, bénévole, professeur de sociologie au Cégep de Chicoutimi

Courriel: jean-marie tremblay@uqac.ca

Site web pédagogique : http://www.uqac.ca/jmt-sociologue/

Dans le cadre de: "Les classiques des sciences sociales" Une bibliothèque numérique fondée et dirigée par Jean-Marie Tremblay, professeur de sociologie au Cégep de Chicoutimi Site web: http://classiques.uqac.ca/

Une collection développée en collaboration avec la Bibliothèque Paul-Émile-Boulet de l'Université du Québec à Chicoutimi Site web: http://bibliotheque.uqac.ca/ 


\section{Politique d'utilisation de la bibliothèque des Classiques}

Toute reproduction et rediffusion de nos fichiers est interdite, même avec la mention de leur provenance, sans l'autorisation formelle, écrite, du fondateur des Classiques des sciences sociales, Jean-Marie Tremblay, sociologue.

Les fichiers des Classiques des sciences sociales ne peuvent sans autorisation formelle:

- être hébergés (en fichier ou page web, en totalité ou en partie) sur un serveur autre que celui des Classiques.

- servir de base de travail à un autre fichier modifié ensuite par tout autre moyen (couleur, police, mise en page, extraits, support, etc...),

Les fichiers (.html, .doc, .pdf., .rtf, .jpg, .gif) disponibles sur le site Les Classiques des sciences sociales sont la propriété des Classiques des sciences sociales, un organisme à but non lucratif composé exclusivement de bénévoles.

Ils sont disponibles pour une utilisation intellectuelle et personnelle et, en aucun cas, commerciale. Toute utilisation à des fins commerciales des fichiers sur ce site est strictement interdite et toute rediffusion est également strictement interdite.

L'accès à notre travail est libre et gratuit à tous les utilisateurs. C'est notre mission.

Jean-Marie Tremblay, sociologue

Fondateur et Président-directeur général, LES CLASSIQUES DES SCIENCES SOCIALES. 
Cette édition électronique a été réalisée par Jean-Marie Tremblay, bénévole, professeur de sociologie au Cégep de Chicoutimi à partir de :

Sylvie FAINZANG

“La construction culturelle de la norme et de la pathologie".

Un article publié dans la revue Confrontation entre science et vivant. Numéro spécial intitulé : "Normal et Pathologique: où se trouve la frontière aujourd'hui?” (Publication du Pôle Universitaire Européen, Montpellier et LanguedocRoussillon), 1999, n¹, pp. 9-12.

[Autorisation formelle accordée par l'auteure le 11 février 2009 de diffuser cette œuvre dans Les Classiques des sciences sociales.]

d. Courriel : sylvie.fainzang@orange.fr

Polices de caractères utilisée :

Pour le texte: Times New Roman, 12 points.

Pour les citations : Times New Roman, 12 points.

Pour les notes de bas de page : Times New Roman, 12 points.

Édition électronique réalisée avec le traitement de textes Microsoft Word 2008 pour Macintosh.

Mise en page sur papier format : LETTRE (US letter), 8.5'’ x 11'’)

Édition numérique réalisée le 31 mars 2009 à Chicoutimi, Ville de Saguenay, province de Québec, Canada.

\section{CFait avec}




\section{Table des matières}

$\underline{\text { Introduction }}$

1. La folie dans une société africaine (les Bisa du Burkina Faso):

2. La stérilité en milieu immigré africain polygamique.

3. L'alcoolisme en France 
Sylvie FAINZANG

“La construction culturelle de la norme et de la pathologie”.

Un article publié dans la revue Confrontation entre science et vivant. Numéro spécial intitulé : "Normal et Pathologique: où se trouve la frontière aujourd'hui?” (Publication du Pôle Universitaire Européen, Montpellier et LanguedocRoussillon), 1999, n¹, pp. 9-12.

\section{INTRODUCTION}

$\underline{\text { Retour à la table des matières }}$

Pour contribuer à la discussion sur le normal et le pathologique, je me propose de présenter ici la perception de la norme et de la pathologie dans des univers culturels divers, et évoquer les conclusions qu'on peut en tirer avec une approche anthropologique.

Je prendrai trois exemples:

1. la folie dans une société africaine

2. la stérilité en milieu immigré africain vivant en ménage polygamique

3. l'alcoolisme en France

Avec ces différents exemples, on verra comment se trouve posée la définition du pathologique dans ses rapports avec la norme, et ce qu'il y a de commun aux différentes sociétés humaines dans leur manière de penser la pathologie. 


\section{1 - La folie dans une société africaine (les Bisa du Burkina Faso):}

\section{$\underline{\text { Retour à la table des matières }}$}

La folie est considérée comme le résultat du désordre subi par un organe, causée par une perturbation (un déplacement ou un renversement) du cerveau ou du foie. L'activité mentale est assumée par ces deux organes, bien que le foie jouisse d'un statut prépondérant en la matière. Le cerveau est le siège de la pensée, le foie est le siège de la raison. Les Bisa reconnaissent deux formes de folie, auxquelles correspond un degré de gravité différent, lié à l'organe affecté.

- la tête/ le cerveau renversé (hallucinations, propos incohérents, incompris, et jugés anormaux)

le foie renversé.

Le traitement de la folie consistera à administrer un remède apte à replacer l'organe affecté dans le bon sens. Ce remède relève de ce qu'on peut appeler l'homéopathie symbolique dans la mesure où il est confectionné à partir d'herbes ayant été entraînées dans les fossés par les pluies, c'est-à-dire par des herbes qui ne sont plus à leur place normale, et qui ont ainsi subi une altération comparable à celle de l'organe affecté dans le cas de la folie: le cerveau ou le foie. Le traitement est donc fondé sur une analogie entre l'état du malade et l'état de l'objet utilisé comme remède.

Si la distinction opérée entre la folie du cerveau et la folie du foie (cerveau renversé et foie renversé) renvoie à une différence de degré, en particulier au degré de gravité (niveau quantitatif de l'appréciation du mal), elle renvoie également à une distinction des comportements que la folie engage, sur le plan qualitatif. On reconnaît un homme atteint de cerveau renversé essentiellement aux propos étranges qu'il tient, à ce qu'il dit: "il dit ce qu'on ne dit pas".

En revanche, la folie entraînée par le renversement du foie, jugée grave, est celle de l'individu qui par exemple détruit la nourriture, brûle les maisons, casse les jarres, viole les femmes, frappe les enfants sans raison, se suicide, etc. La distinction entre ces deux types de folie relèvent respectivement de l'ordre du geste et 
de l'ordre du verbe. Le caractère de gravité accordée à la folie gestuelle viendrait peut-être de ce qu'elle est rapprochée de la violence de l'animalité. Avec le foie renversé, c'est l'animalité qui fait rage. Si le fait de détruire les biens et de porter atteinte au groupe est particulièrement patent dans cette forme de folie, il ne constitue pas le seul critère de détermination de sa gravité. Car enfin il pourrait être jugé tout aussi dangereux pour le groupe que certaines choses soient dites.

La comparaison entre les organes affectés dans le cas de ces deux maladies laisse apparaître une différence notoire au niveau du jugement porté sur le comportement du fou. Avec le foie renversé, la raison du patient est altérée, tandis qu'avec le cerveau renversé, seule sa pensée est perturbée, sa raison est intacte. Avoir la maladie du cerveau renversé, c'est explicitement avoir des pensées qui ne sont plus conformes à la pensée dominante. Mais on peut dire ce que les autres ne disent pas et avoir ses raisons de le dire. Le cerveau renversé marque donc le cadre de la pensée subversive. Le traitement est destiné à rendre sa pensée conforme, à la normaliser. Diagnostiquer chez quelqu'un la maladie du cerveau renversé, c'est lui reconnaître ses raisons de dire ce qu'il dit, mais lui en refuser le droit.

La folie peut aussi être interprétée comme un signe d'élection, visant à faire passer le fou dans le camp des devins, une fonction sociale de toute première importance chez les Bisa, puisque ce sont eux qui sont chargés de diagnostiquer les maladies et de résoudre les désordres mettant en péril la reproduction sociale. Ils sont considérés comme les intermédiaires entre les hommes et les génies, ces puissances surnaturelles supposées régir la société des hommes.

La pathologie que constitue la folie d'élection marque cependant toujours, elle aussi, un écart par rapport à une norme.

L'irruption de la folie d'élection est tout particulièrement manifestée à travers la perte d'une personne (le futur devin) en brousse. La mise en parallèle entre la notion de folie et la notion de perte en brousse (qui est le monde des génies), situe le futur devin à l'écart de l'espace normal des hommes (le village). L'éloignement spatial fournit la matière d'une métaphore sur la folie où l'égarement physique est la condition de l'égarement mental. Cette conception n'est d'ailleurs pas étrangère à d'autres systèmes de représentations, et on peut la trouver exprimée sous la plume de Michel Foucault dans l'emploi du terme "égaré" pour signifier le fou. 
L'égarement en brousse est l'inscription dans l'espace d'une rupture avec le monde de la norme humaine: le village.

La différence entre le fou-malade et le fou-élu, est que ce dernier, le devin, n'a pas le cerveau ni le foie renversé, mais au contraire, il a, dit-on, beaucoup de foie. La possession, en grande quantité, de foie, rend apte au travail particulièrement difficile du devin, celui de voir ce que les autres hommes ne voient pas, à savoir l'intérieur des choses. Il est un homme hors du commun, et sa cécité aux choses ordinaires, normalement vues par les autres hommes (le devin est appelé bu l'aveugle) exprime sa clairvoyance mentale et son aptitude à ordonner aux autres hommes les conduites à tenir, en toute conscience (le foie est aussi le siège de la conscience), en vue d'assurer la reproduction sociale.

\section{2 - La stérilité en milieu immigré africain polygamique.}

$\underline{\text { Retour à la table des matières }}$

Le vécu en ménage polygamique est fortement conflictuel et donne lieu à de fortes relations de rivalités entre co-épouses. Or il faut savoir que la reproduction biologique est un enjeu fondamental pour les femmes dans les sociétés dont sont originaires les familles africaines immigrées en France. Elle est un vecteur de prestige et de reconnaissance sociale et détermine le statut social et conjugal des femmes. Les femmes ne sont ainsi socialement reconnues et valorisées qu'en fonction de leur aptitude à procréer.

Etant donné les fortes rivalités engendrées structurellement par l'institution polygamique, les femmes se livrent à une véritable compétition en matière de production d'enfants. La nécessité dans laquelle elles sont de dépasser leur coépouse en ce qui concerne le nombre d'enfants qu'elles mettront au monde, les conduisent à craindre que leur co-épouse ne cherche à les rendre stérile, et les incitent à tenter, par toutes sortes de procédés rituels, à rendre leur co-épouse stérile lorsque c'est son tour de partager la couche du mari (système de tours qui règle le partage des services domestiques et sexuels des femmes). Tous les problèmes relatifs à la sexualité et à la reproduction (c'est-à-dire à ce par quoi les fem- 
mes sont socialement définies), sont expliqués par le maraboutage (c'est-à-dire à l'action magique, protectrice ou persécutrice, accomplie par un marabout = détenteur de la connaissance coranique). Dès lors, la stérilité est inévitablement considérée comme le résultat de l'action persécutrice de la co-épouse, et une femme atteinte de stérilité se rendra presque toujours chez un marabout, jugé apte à neutraliser la persécution de la co-épouse, de préférence à une consultation de stérilité à l'hôpital.

La crainte du maraboutage sera encore plus forte si la victime est fondée à croire que sa co-épouse a de bonnes raisons de lui en vouloir et elle existe également lorsque les co-épouses ne vivent pas ensemble: lorsque, par exemple, une seconde épouse a indûment pris la place de la première, en venant en France seule avec son mari, tandis que la première a été laissée au pays. Pourquoi? Parce que la première épouse est considérée comme la plus légitime, puisqu'elle a été choisie par la famille et que son union avec le mari scelle une alliance entre les familles respectives des époux, contrairement aux épouses suivantes qui peuvent être choisies par le mari sans que la famille n'intervienne nécessairement. Plus légitime, la première femme a un certain nombre de prérogatives, en vertu desquelles elle devrait, normalement, être emmenée la première, au cas où le mari ne pourrait emmener toutes ses femmes avec lui. Dans le cas de maladie de la seconde épouse, stérilité ou autre, il sera tout naturel d'en trouver la cause dans la transgression de cette norme sociale. La pathologie est ici le résultat et le signe d'un désordre social.

\section{3 - L'alcoolisme en France}

\section{$\underline{\text { Retour à la table des matières }}$}

Pour l'anthropologue, s'interroger sur la frontière entre norme et pathologie en matière d'alcoolisme, autrement dit sur la frontière entre consommation normale et consommation pathologique, c'est tenter de cerner ce qui définit et ce qui caractérise l'alcoolique tant aux yeux des autres qu'aux yeux des anciens buveurs euxmêmes. (La question de la définition et de la représentation de soi comme alcoolique, et donc comme relevant de la pathologie, est tout à fait décisive dans la me- 
sure où cette perception constitue souvent l'événement moteur du vouloir-guérir, et joue donc un rôle déterminant sur l'avenir social et thérapeutique de l'individu).

L'alcoolique est celui qui a perdu ses capacités humaines, une perte exprimée à travers son corps, et notamment à travers le tremblement de ses membres: l'impossibilité de se lever ou le fait de tomber marque l'état d'un être diminué, incapable de garder la station debout, considérée comme celle de l'homme en pleine possession de ses capacités. Assimilé au vieillard grabataire ou au petit enfant dépendant de sa mère, incapable de s'habiller tout seul, l'alcoolique est celui qui s'est éloigné du modèle de l'individu autonome et accompli.

L'alcoolique est aussi celui qui a perdu ses capacités morales ou ses qualités personnelles et le sens des valeurs, et dont le caractère est vu comme le résultat d'une inversion de celui qu'il avait naturellement avant, une inversion qui est exprimée par la parabole de Dr Jekyll et Mr Hyde, une nature positive transformée en nature négative. Il est celui qui est devenu agressif, violent, brutal, avec un comportement qui le situe aux confins de l'animalité (sa violence est d'ailleurs souvent exprimée au moyen de termes habituellement utilisés pour décrire les comportements animaux: on parle de sa sauvagerie, de sa férocité, de son comportement déchaîné).

Toutefois, l'animalisation de l'alcoolique ne se fait pas que par référence à sa violence. Elle peut procéder d'une infériorisation, liée au fait que le conjoint perçoit désormais le buveur comme un être déchu de sa dignité d'homme, infériorisation également exprimée à travers le langage de l'infantilisation.

L'alcoolique peut au contraire être caractérisé par son incapacité à assumer son rôle social et par son caractère passif et apathique, l'associant au règne végétal. Dans tous les cas, le comportement social de l'alcoolique est la première chose mise en avant. Rattaché soit à un autre âge (biologique et social), soit à un autre règne (animal ou végétal), l'alcoolique est celui qui a perdu les caractères et les acquis de l'humanité. Le passage dans l'univers de la pathologie correspond à la perte de son humanité. 
Le but de mon propos n'est pas seulement de dire qu'il n'y a pas de norme absolue ni de pathologie absolue, et d'inscrire notre discussion dans un relativisme effréné. Il est aussi de souligner que la désignation comme normal et pathologique ne se fait pas hors d'une organisation sociale et qu'elle ne prend sens qu'en fonction de ce contexte. La perception de la frontière entre normal et pathologique répond à un système de représentations donné. Dans certains cas, c'est la situation qui est avant tout perçue comme pathologique (ex. $2^{\text {ème }}$ femme avant $1^{\text {ère }}$ polygamique) et non le corps, et qui doit être réparée. La réparation est donc conçue comme devant être tout autant sociale (sinon plus) que biologique.

Si le pathologique fut à tort, comme l'a montré Canguilhem, qualifié comme relevant de l'ordre de l'excès ou du défaut dans certaines théories médicales passées, on peut néanmoins reconnaître qu'entre le normal et le pathologique, la pensée humaine perçoit des différences de degré. Or c'est bien cette pensée humaine que l'anthropologie a la charge d'analyser. En effet, il arrive que les critères de l'excès ou du défaut permettent aux individus ou aux sociétés, de conclure à la présence du pathologique. La représentation de la pathologie en termes de degré est donc une représentation commune, comme l'atteste l'idée de démesure fréquemment associée à l'alcoolisme (ou l'idée d'une grande quantité de foie attribuée au devin). Mais en même temps, cette démesure introduit à l'existence d'autre chose qualitativement: l'alcoolique n'est pas seulement un individu qui boit plus ou trop, il devient ontologiquement autre; il sort du cadre de ce qui définit l'humain ou l'humanité, de même que le devin (le fou-élu) n'est pas seulement quelqu'un qui a beaucoup de foie, il sort du groupe des hommes ordinaires pour devenir qualitativement autre: un être intermédiaire entre les hommes et les génies.

On s'aperçoit donc, à l'analyse, que cette différence quantitative renvoie en même temps à une différence qualitative et l'engendre. Le pathologique désigne alors quelque chose de fondamentalement différent (dévié, déformé, autre, comme avec le cas du foie renversé) introduisant au désordre.

La frontière entre le normal et le pathologique frontière renvoie à d'autres frontières auxquelles elle se superpose souvent, comme celle existant entre les notions de santé et de maladie bien sûr, mais aussi de pureté et d'impureté, ou d'ordre et de désordre, autant de couples d'opposition dont chacun des termes ne prend sens que par rapport à l'autre. 
Cette frontière est mouvante. Elle fluctue non seulement dans l'espace, d'une culture à une autre, mais aussi dans le temps, d'une période à une autre. Et puisqu'il s'agit d'un problème de frontières, il est, en tant que tel (et comme toutes les frontières), sujet à des fluctuations, des revendications, des dénégations au sujet de zones déclarées appartenir à un camp ou un autre.

L'indécision de cette frontière fait que ce qui est franchement à la marge en un temps ou en un lieu donnés peut être au seuil en un autre; aussi, pour rendre compte de cette mouvance de la frontière entre normal et pathologique, faut-il peut-être distinguer entre liminarité et marginalité, c'est-à-dire entre ce qui est tout près d'être intégré à la norme et ce qui totalement situé en marge de cette norme, l'une et l'autre, pouvant dans des contextes sociaux et historiques différents, passer aisément d'un état à un autre.

On pourra rétorquer que la pathologie est d'une certaine façon objectivable, puisqu'elle peut signifier ou désigner la modification de l'état d'une fonction ou d'un organe. Mais le point de vue de l'anthropologue est d'envisager cette définition comme l'objet d'une construction culturelle, tout comme celle de maladie d'ailleurs.

Cette guerre de frontière que pose la problématique du normal et du pathologique ne peut être tranchée par une autorité suprême qui serait la bio-médecine. Car pour l'anthropologue, la bio-médecine est un système de pensée comme un autre, face aux différents systèmes culturels à l'intérieur desquels est pensée la frontière entre le normal et le pathologique. La bio-médecine est donc pour les anthropologues un univers culturel supplémentaire avec sa logique propre, mais non doté d'une compétence supérieure.

A cet égard, un des principaux apports des travaux en sciences sociales sur la douleur est d'avoir montré que la douleur n'était pas seulement reconnue comme le signe d'une pathologie, mais qu'elle se voyait aujourd'hui accorder le statut même de pathologie, phénomène qui est un produit de la société contemporaine.

La contribution que l'anthropologie peut apporter à la bio-médecine n'a donc pas pour but de déterminer en quoi les autres systèmes se tromperaient, mais de l'aider à comprendre à quelle(s) logique(s) renvoie l'établissement de cette frontière dans les différentes cultures et chez les individus qui en relèvent. 